Physics of Fluids

August 2004; 16(8) : 3079-3085

http://dx.doi.org/10.1063/1.1767543

(c)2005 American Institute of Physics
Archimer http://www.ifremer.fr/docelec/ Archive Institutionnelle de l'Ifremer

\title{
Vortex erosion in a shallow-water model
}

\author{
S. Louazel ${ }^{1^{*}}$, B. L. Hua ${ }^{2}$ \\ ${ }^{1}$ Centre Militaire d'Océanographie, EPSHOM, 13 rue du Chatellier, 29609 Brest, France \\ ${ }^{2}$ Laboratoire de Physique des Océans, IFREMER, Boîte Postale 70, 29280 Plouzané, France \\ *: Corresponding author : louazel@shom.fr
}

\begin{abstract}
The purpose of this paper is to study the erosion of eddies by filamentation in a shallowwater model where both fast and slow components of the dynamics coexist. In presence of inertiagravity waves this filamentation process is indeed found to be altered. However, the information required to analyze the filamentation process is shown to be entirely captured by the slow component of the flow and regions of filament ejection can be identified from a diagnostic criterion based on instantaneous slow quantities. This is illustrated numerically in a reduced gravity model for an initial elliptic vortex of distributed potential vorticity in presence of Poincaré waves.
\end{abstract}

Keywords: Physics, fluids \& plasmas, mechanics 


\section{Introduction}

Large-scale geophysical flows are characterized by coherent vortices where enstrophy is concentrated. The filamentation of these structures, a manifestation of the enstrophy cascade, has been largely studied for two-dimensional (2D) incompressible flows. Some authors investigate, theoretically $\left(\mathrm{Kida}^{1}\right)$ or numerically (Mariotti et $a .^{2}{ }^{2}$ ), the vortex evolution in an external shear flow. Under the influence of the shear, the vortex is constrained to expell vorticity filaments in order to maintain its coherence. Filaments produced by this mechanism can roll up in new vortices depending on their stability (Dritschel et al. ${ }^{3}$ ). Vortex filamentation can also be produced under the own vortex deformation field as analyzed by Melander et al. ${ }^{4}$, Polvani et $a l .{ }^{5}$ or Kimura and Herring. ${ }^{6}$ The objective of this paper is to study the evolution of an elliptic vortex in a shallow-water (hereafter SW) context.

The wave-vortex interaction has been studied in many different manners (theoretically or numerically, in spectral space or in physical space,etc.). In the case of nondissipative waves $^{7,8,9}$, all studies find that there is no significant energy transfer between the fast and the slow components of the flow in the small Rossby number limit. However, waves can modify the vortical component of the flow and Bühler and McIntyre ${ }^{10}$ insist on the irreversible modification of potential vorticity (hereafter PV) contours advection by waves which is "qualitatively different from that expected from a naive consideration of Stokes drifts alone". In the case of dissipating or breaking waves, PV can be created as illustrated in shallow-water flow by Bühler. ${ }^{11}$

The process of filamentation is associated with PV gradient intensification. The evolution of PV gradient has been examined by $\mathrm{Okubo}^{12}$ and Weiss ${ }^{13}$ in a context where the velocity gradient tensor is considered as slowly variable along the Lagrangian trajectories, its real eigenvalues corresponding to areas of filamentation. Because it is simple and based on instantaneous quantities, this criterion is widely applied to study the process of filamentation. Carton et al. ${ }^{14}$ estimate it from hydrological data to localize warm and salty filaments near Meddies (Mediterranean water Eddies). Maassen et al. ${ }^{15}$ estimate it 
from laboratory data to study the detachment of viscous boundary layers. It should be pointed out that data from geophysical or laboratory flows are often available as instantaneous snapshots and their study requires instantaneous criteria. Herbette et al. ${ }^{16}$ also use the Okubo-Weiss criterion, but in shallow-water numerical experiments, to identify the filamentation areas of a surface vortex as it encounters a seamount.

Thus, although it does not consider any time variation of the velocity gradient tensor, the Okubo-Weiss criterion is largely used even in experiments involving vortices whose edge is a region where these variations are important as noted by Hua and Klein ${ }^{17}$ who instead study the eigenvalues of the acceleration gradient tensor which governs the second order time derivative of the PV gradient. For 2D quasigeostrophic flows, the approach of Lapeyre et al..$^{18}$ and Klein et $a l^{19}$ is based on the investigation of the PV gradient alignment in strain coordinates. Their criterion is related to the Okubo-Weiss criterion, it is also estimated from instantaneous quantities but takes furthermore into account the rotation of the strain principal axes which depends on Lagrangian accelerations, thus on the balance of forces. This criterion is more appropriate to our study of vortex filamentation than the Okubo-Weiss one since it is this rotation of the strain axes which is important on the vortex edge.

Note that this criterion is not only used to study filamentation but also for perturbation growth on a basic state flow. Indeed the mathematical expressions of vector stretching dynamics in the evolution equations of PV gradient and velocity perturbation are similar. Rivière $e t a l .{ }^{20}$ use the criterion of Lapeyre et $a l .{ }^{18}$ in a quasigeostrophic framework and Straub $^{21}$ tests it in a primitive equations model where it is estimated from the basic flow which only has a slow component.

The Lapeyre et al. ${ }^{18}$ criterion is more accurate than the Okubo-Weiss one but is based on restrictive asumptions such as the slowness of the strain axes rotation. Its advantage is to provide a diagnostic criterion based on instantaneous quantities since data over a long time can only be available in numerical experiments but not in geophysical or laboratory datasets. A more complete approach is to consider the full Lagrangian history on which the process of filamentation depends (cf. Haller, ${ }^{22}$ who has developed 
a Lagrangian version of the Okubo-Weiss criterion to localize hyperbolic and elliptic structures and the application of the method within the context of the polar vortex flow by Koh and Legras ${ }^{23}$ ).

This paper that aims at analyzing the filamentation process of a vortex in SW dynamics is organized as follows. In Sec. II, the similarities and differences of this process with or without inertia-gravity (hereafter IG) waves are assessed from numerical SW experiments. In spite of its limitations, we attempt to use the Lapeyre et al. ${ }^{18}$ criterion to localize the areas of filament ejection but the presence of rapid motions causes the criterion to fail. Thus in Sec. III, the filamentation process is diagnosed from the slow component of the flow. Finally, results are summarized and discussed in Sec. IV.

\section{PV gradient growth in shallow-water (SW)}

We consider a simplified version of the primitive equations model MICOM (Miami Isopycnic Coordinate Ocean Model) on an /it $\mathrm{f}$ plane. The vertically discretized equations in isopycnal coordinates (Bleck and Boudra ${ }^{24}$ ) reduce to the SW equations. In a square doubly periodic domain, we use a reduced gravity configuration without any diapycnal mixing,

$$
\begin{aligned}
& \frac{D \boldsymbol{u}}{D t}+f \boldsymbol{k} \wedge \boldsymbol{u}=-g^{\prime} \nabla h, \\
& \frac{\partial h}{\partial t}+\nabla \cdot((H+h) \boldsymbol{u})=0,
\end{aligned}
$$

where $\boldsymbol{u}=(u, v)$ represents the horizontal velocity field, $H$ the layer depth at rest, $h$

the height fluctuation, $\frac{D}{D t}=\frac{\partial}{\partial t}+\boldsymbol{u} . \nabla$ the Lagrangian derivative, $\boldsymbol{k}$ the vertical unitary vector, and $g^{\prime}$ the reduced gravity parameter. For numerical reasons, isopycnal diffusion of momentum is added to the first equation.

In SW dynamics the key quantity is PV which is a Lagrangian invariant, the PV 
anomaly is denoted by $q$,

$$
q \equiv H\left(\frac{\omega+f}{H+h}-\frac{f}{H}\right)=\frac{\omega-f \frac{h}{H}}{1+\frac{h}{H}}
$$

where $\omega=\partial_{x} v-\partial_{y} u$ represents the relative vorticity. Moreover, we will note hereafter $\sigma_{n}=\partial_{x} u-\partial_{y} v$ the normal strain, $\sigma_{s}=\partial_{x} v+\partial_{y} u$ the shear strain, $\sigma=\sqrt{\sigma_{n}^{2}+\sigma_{s}^{2}}$ the strain rate and, $\delta=\partial_{x} u+\partial_{y} v$ the divergence.

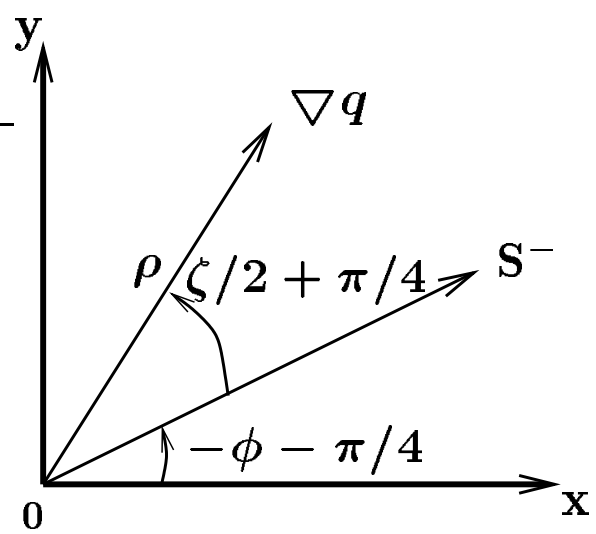

Figure 1: Magnitude $\rho$ of PV gradient and angles defining the directions of the strain compressional axis $\boldsymbol{S}^{-}$and PV gradient.

\section{A. Criterion of PV gradient growth}

The filamentation process is associated with PV gradient intensification and from the dynamics of PV gradient, different diagnostic criterions can be derived to study this process. Considering PV conservation along a Lagrangian trajectory, which reduces to $\frac{D q}{D t}=0$ on the $/$ it $\mathrm{f}$ plane, the evolution equation of $q$ gradient is obtained,

$$
\frac{D \nabla q}{D t}=-[\nabla \boldsymbol{u}]^{*} \nabla q
$$

where $[\nabla \mathbf{u}]^{*}$ represents the transpose of the velocity gradient tensor.

The approach of $\mathrm{Okubo}^{12}$ and Weiss ${ }^{13}$ consists in considering in (3) that $-[\nabla \boldsymbol{u}]^{*}$ is slowly varying along the Lagrangian trajectories, hence they consider its eigenvalues 
which are $\left(-\delta \pm \sqrt{\sigma^{2}-\omega^{2}}\right) / 2$. Thus for nondivergent flows, the physical space is divided into rotation dominated areas $(|\omega|>\sigma)$ and strain dominated areas $(\sigma>|\omega|)$ where an exponential growth of PV gradient norm is expected. Note that, even for small divergent flows, the widely used Okubo-Weiss quantity is $\sigma^{2}-\omega^{2}$.

For $2 \mathrm{D}$ motions, Lapeyre et al. ${ }^{18}$ improve the above criterion by taking into account the strain variations along the Lagrangian trajectories through the rotation of its principal axes. This criterion is developed here for SW equations. As the approach is based on the investigation of the $q$ gradient alignment in strain coordinates, $q$ gradient and the strain rates are written in terms of magnitude and angles,

$$
\nabla q=\rho\left[\cos \left(\frac{\zeta}{2}+\frac{\pi}{4}\right), \sin \left(\frac{\zeta}{2}+\frac{\pi}{4}\right)\right], \quad\left(\sigma_{s}, \sigma_{n}\right)=\sigma[\cos (2 \phi), \sin (2 \phi)]
$$

where the angle $\phi$ defining the direction of the strain compressional axis, the norm $\rho$ of $q$ gradient and the angle $\zeta$ characterizing its position relatively to the compressional axis are illustrated in Fig. 1. Dritschel et $a .^{3}{ }^{3}$ use the same formalism to investigate the stability of a vorticity filament under the action of strain.

Then the vector equation (3) is split into two scalar equations, one for $\rho$ and one for $\zeta$,

$$
\begin{gathered}
\frac{1}{\rho^{2}} \frac{D \rho}{D t}=-\sigma \sin \zeta-\delta, \\
\frac{D \zeta}{D t}=\sigma(r-\cos \zeta),
\end{gathered}
$$

where $r$ compares the strain to an "effective rotation" which is the difference between the relative vorticity and the rotation rate $-2(D \phi / D t)$ of the strain axes,

$$
r \equiv \frac{\omega+2(D \phi / D t)}{\sigma}
$$

The SW expression of $2(D \phi / D t)$, which depends on the spatial derivatives of Lagrangian 
accelerations, is the following:

$$
\begin{gathered}
2 \frac{D \phi}{D t}=\frac{\sigma_{s}\left(D \sigma_{n} / D t\right)-\sigma_{n}\left(D \sigma_{s} / D t\right)}{\sigma^{2}} \\
\frac{D \sigma_{n}}{D t}=\partial_{x}\left(\frac{D u}{D t}\right)-\partial_{y}\left(\frac{D v}{D t}\right)-\delta \sigma_{n} \quad, \quad \frac{D \sigma_{s}}{D t}=\partial_{y}\left(\frac{D u}{D t}\right)+\partial_{x}\left(\frac{D v}{D t}\right)-\delta \sigma_{s} .
\end{gathered}
$$

Note that the divergence $\delta$, which is zero in the 2D framework of Lapeyre et al. ${ }^{18}$, is directly involved in the magnitude equation (5) and indirectly in the orientation one (6) via the expression of the rotation of the strain principal axes (8). All quantities can be obtained diagnostically at each instant.

Assuming that $r$ is slowly varying along Lagrangian trajectories, Eq. (6) shows that in the strain dominated regions where $|r|<1$, the vorticity gradient lines up with a specific direction relative to the strain axes such that $\zeta=\zeta_{-}=-\arccos (r)$; according to Eq. (5), for small divergent flows, this alignment is associated with an exponential growth of $\rho$. On the contrary, in the "effective rotation" dominated regions where $|r|>1$, the vorticity gradient rotates and no exponential growth of its magnitude is expected, the vorticity gradient dynamics are more precisely investigated by Klein et al. ${ }^{19}$ Thus $r$ is a criterion, depending on the Lagrangian accelerations (and therefore on forces) via the rotation rate of the strain principal axes, to identify areas of PV gradient intensification.

\section{B. Initialization and experiments}

Two numerical experiments (hereafter denoted by 1 and 2) are performed using the above code at a resolution of $1024 \times 1024$, the Coriolis parameter $f$ is equal to $7.10^{-5} s^{-1}, g^{\prime}$ to $5.10^{-3} \mathrm{~m} . \mathrm{s}^{-2}, H$ to $1000 \mathrm{~m}$ and the domain size to $768 * 768 \mathrm{~km}^{2}$. The Ross by deformation

radius $R=\sqrt{g^{\prime} H} / f$ is equal to $32 \mathrm{~km}$, it is well resolved since the grid size $\Delta$ is such that $R \simeq 40 \Delta$.

A gesotrophic vortex alone is initialized in experiment 1 and a geostrophic vortex and IG waves are initialized in experiment 2 such that the initial PV fields are identical for both experiments. Specifically, in experiment 2, the initial vortex velocity and height 
fields are obtained by PV inversion (see sec. III A) of the following elliptic distributed profile $^{4,6}$ :

$$
\begin{aligned}
& q(l)=q_{0}\left[1-\exp \left\{-C \frac{R_{0}}{l} \exp \left(-\frac{R_{0}}{R_{0}-l}\right)\right\}\right] \text { for } 0 \leq l<R_{0}, \\
& q(l)=0 \text { for } l \geq R_{0}, \\
& l=\sqrt{a x^{2}+b y^{2}}, \\
& q_{0}=-3.10^{-5} s^{-1} \quad a=1 \quad b=10 \quad R_{0}=6 R \quad C=2.5608517 .
\end{aligned}
$$

Since PV is a non-linear quantity, the presence of IG waves in this experiment 2 makes the actual initial PV profile $\tilde{q}$ slightly different from (9). In experiment 1 , the initial vortex velocity and height fields are obtained by $\tilde{q}$ inversion.

For both experiments, the height fluctuation $h$ at the vortex center is about $1 / 3 H$, the rotation time scale of such vortex is around $40 * 2 \pi / f$. In experiment 1 , the Rossby and Froude numbers are, respectively, equal to 0.25 and 0.15 . In experiment 2, the Poincaré waves amplitude is about $1 / 30 \mathrm{H}$, their wave lengths are $12 R$ and $8 R$ and the two components are out of phase, the Rossby and Froude numbers are, respectively, equal to 0.25 and 0.3 , specifically the ratio $|\boldsymbol{u}|_{\text {slow }} /|\boldsymbol{u}|_{\text {fast }}$ is about 0.5 .

\section{Filamentation process}

In both experiments, the vortex shows some similar behaviors. As studied in 2D flows by Melander et $a l^{4}$ and Kimura and Herring, ${ }^{6}$ the vortex relaxes towards axisymmetry as the result of filament ejection. Indeed the ellipticity of the vortex which is initially equal to 0.95 progressively decreases to reach 0.6 after two vortex rotation time scales. The filamentation process occurs through successive stages. We will focus hereafter on the first two sheddings of such filaments, the primary one occurring at the tip of the longest axis of the ellipse after around a quarter vortex rotation time scale and the secondary one that is more laterally located occurring after a full vortex rotation time scale.

Whereas the first shedding occurs in the same way and at the same position for both vortices, for the second one the shape and the localization of PV filaments are 
different (cf. boxes of Fig. 2). There is a shift in the position of the ejection points of the secondary filaments in presence of IG waves (the distance between the primary and secondary ejection points changes by a factor of 1.2 between the two experiments).

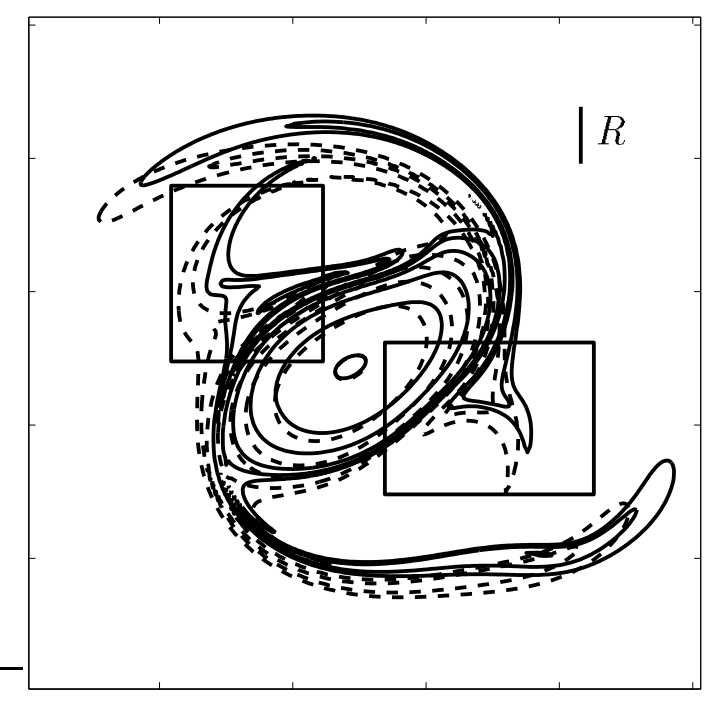

Figure 2: PV anomaly field after a vortex rotation time scale, solid contours are for experiment 2 and dashed ones for experiment 1 . All contours are negative, contour interval is $6.10^{-6} s^{-1}$. The scale of the Rossby deformation radius $R$ is given.

Pursuing further in time the integration of both experiments, we have found that differences in filamentation were irreversible, as already noted by Bühler and McIntyre. ${ }^{10}$ We have used as an index for measuring this difference the quantity $<\left|q_{1}-q_{2}\right|>/<\left|q_{1}\right|>$, where $q_{1}\left(q_{2}\right)$ represents PV anomaly of experiment $1(2)$ and $<$. $>$ the spatial domain average. The nonoscillatory component of this index indeed increases monotonously [Fig. $3(\mathrm{a})]$.

Although the process of filamentation is altered in presence of IG waves, we would like to be able to localize theoretically at a given time the areas of filament ejection, relying on previously established criteria, such as the $r$ criterion. We will use instead of $r$, which involves a division by the strain $\sigma$ that can be zero at some points of the flow, the following $\tilde{r}$ quantity:

$$
\tilde{r}=\frac{\sigma^{3}}{f^{3}}(1-|r|)=\frac{1}{f^{3}}\left[\sigma^{3}-\left|\omega \sigma^{2}+\sigma_{s}\left(D \sigma_{n} / D t\right)-\sigma_{n}\left(D \sigma_{s} / D t\right)\right|\right] .
$$




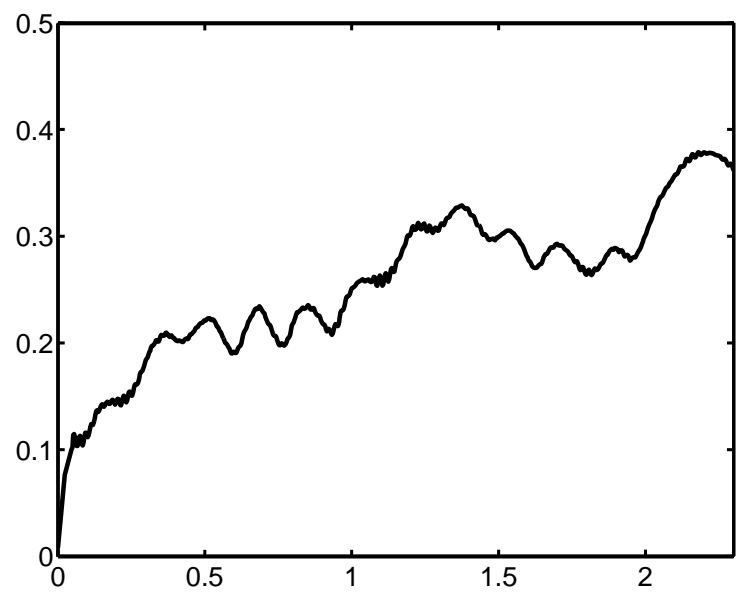

(a)

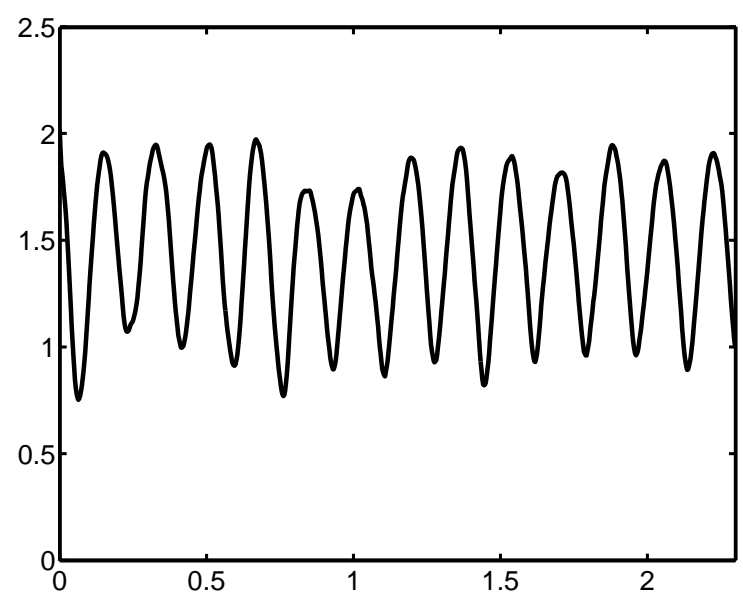

(b)

Figure 3: (a) Time evolution of $<\left|q_{1}-q_{2}\right|>/<\left|q_{1}\right|>$ (b) time evolution of the ratio between the spatial averages of IG strain and geostrophic strain for experiment 2 , the two strains are averaged over a box enclosing $90 \%$ of geostrophic strain. Time is adimensionalized by the vortex rotation time scale.

Therefore, filaments should be ejected where $\tilde{r}>0 . \tilde{r}$ is tested for experiment 1 (cf. Appendix), as it proves to be successful in this case, it is also plotted in Fig. 4(a) for experiment 2: $\tilde{r}$ is obviously no longer relevant. For primitive equations, the existence of rapid inertia-gravity motions introduces fast time scales in the $r$ criterion. Indeed, the rotation of the strain axes $2(D \phi / D t)$, which appears in the $r$ quantity $(7)$ and which depends on the Lagrangian accelerations, takes large instantaneous values. Herbette et $a l .{ }^{16}$ note that the presence of IG waves in their experiments prevents them from directly using this criterion to study the filamentation of a vortex that encounters a seamount in a SW context. IG waves have a significant strain field: Fig. 3(b) shows the time evolution of the ratio between the spatial averages of IG strain and geostrophic strain, the two strains are averaged over a box enclosing $90 \%$ of geostrophic strain for the whole simulation. This figure illustrates that the IG strain is of the same order as geostrophic strain, while PV dynamics has a mostly slow component. This suggests that IG strain should not be taken into account in the evaluation of $r$ for analyzing PV filamentation, and that one should filter IG waves before using an appropriate instantaneous $r$ criterion. The above statements only hold for the special case of the tracer PV and do not apply for passive tracer fields that are not correlated with the slow velocity field. 


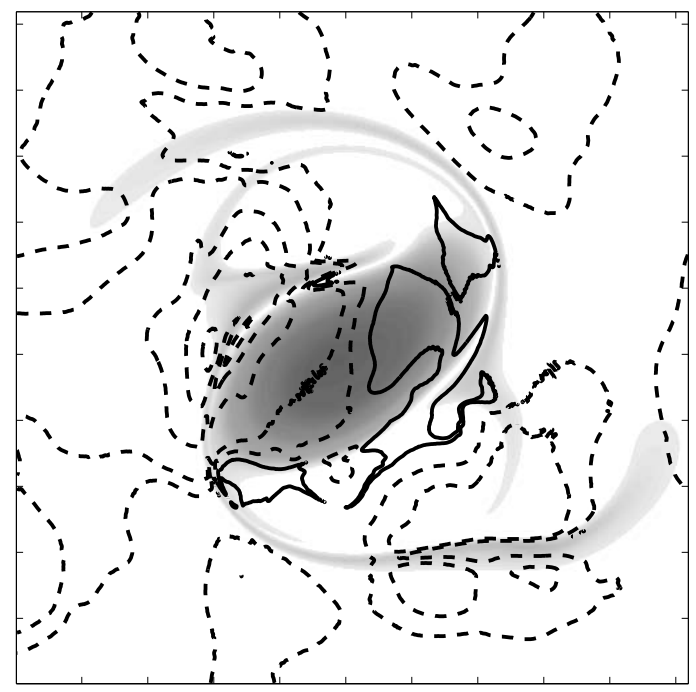

(a)
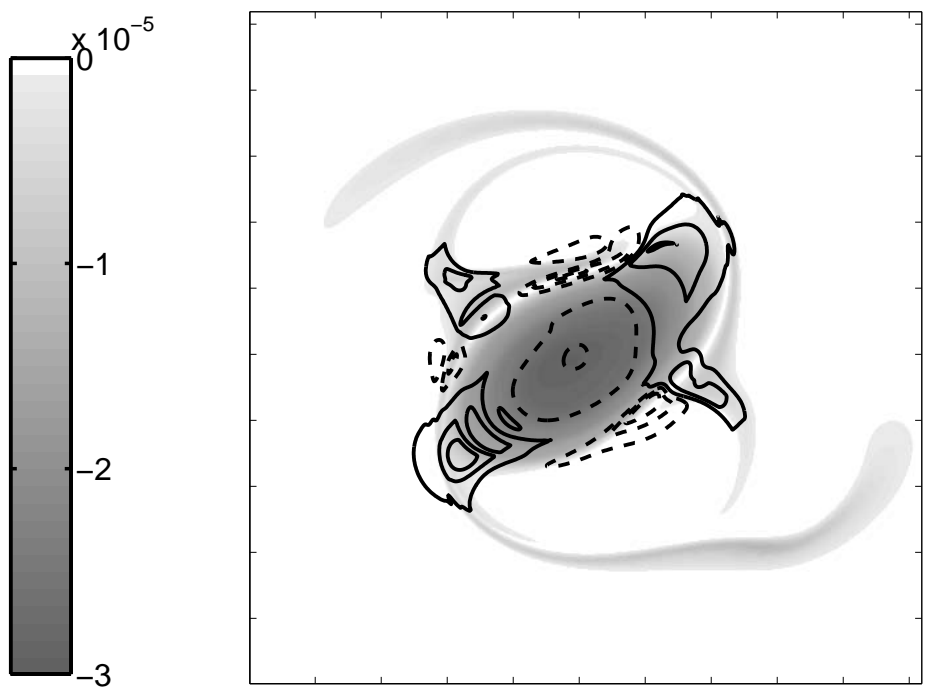

(b)

Figure 4: (a) $\tilde{r}$ field (b) $\tilde{r_{0}}$ field after a vortex rotation time scale of experiment 2 . For both figures, solid lines are positive contours $\left[2.10^{-4}\right.$ for (a), interval is $10^{-3}$ for (b)] and dashed lines are negative contours [interval is $2.10^{-2}$ for (a) and $10^{-3}$ for (b)], the gray shading corresponds to the PV anomaly field.

\section{Slow motion extraction}

\section{A. Filtering from geostrophic balance}

The filtering can be done by PV inversion. A set of diagnostic equations describing the slow component of the flow is necessary for the inversion. Many papers deal with this set of diagnostic equations; indeed in addition of the papers discussing PV inversion $\left(\right.$ Vallis $^{25}$, McIntyre and Norton ${ }^{26}$, Mohebalhojeh ${ }^{27}$ ), these equations are involved in balanced models (Warn et al. ${ }^{28}$, Muraki et al. ${ }^{29}$, Mohebalhojeh and Dritschel ${ }^{30}$ ) which furthermore use a prognostic equation for the evolution of the slow component.

A first approximation consists in finding the geostrophic flow, characterized by a stream function $\psi_{0}$, by inverting the exact SW PV anomaly $q$ (without expanding it in Rossby number Ro) such that

$$
\frac{\nabla^{2} \psi_{0}-\frac{f^{2} \psi_{0}}{g^{\prime} H}}{1+\frac{f \psi_{0}}{g^{\prime} H}}=q
$$


The corresponding geostrophic flow is

$$
\begin{cases}\boldsymbol{u}_{0}=\boldsymbol{k} \wedge \nabla \psi_{0} & \omega_{0}=\nabla^{2} \psi_{0} \\ h_{0}=\frac{f \psi_{0}}{g^{\prime}} & \delta_{0}=0\end{cases}
$$

All "slow" quantities are noted with the subscript 0.

To estimate the slow criterion $\tilde{r_{0}}$, the strain axes rotation related to the above $\boldsymbol{u}_{0}$ field must be evaluated, according to (8) the corresponding Lagrangian accelerations are required, as $\boldsymbol{u}_{0}$ is $\mathcal{O}\left(R o^{0}\right)$, the accelerations are $\mathcal{O}(R o)$,

$$
\frac{D_{0} \boldsymbol{u}_{0}}{D t} \equiv \frac{\partial \boldsymbol{u}_{0}}{\partial t}+\boldsymbol{u}_{0} \cdot \nabla \boldsymbol{u}_{0} \equiv-\nabla P-f \boldsymbol{k} \wedge \nabla \chi
$$

$P$ and $\chi$ respectively represent the ageostrophic pressure and the divergent part of the slow motion ageostrophic velocity (Hua et al. ${ }^{31}$ ), they are obtained by a $\mathcal{O}(R o)$ expansion of Euler equations,

$$
\begin{aligned}
& \nabla^{2} P=2 J\left(u_{0}, v_{0}\right), \\
& \left(g^{\prime} H \nabla^{2}-f^{2}\right) \nabla^{2} \chi=\nabla \cdot\left(f \omega_{0} \boldsymbol{u}_{0}\right) .
\end{aligned}
$$

From $D_{0} \boldsymbol{u}_{0} / D t$, the rotation rate of the strain axes related to the velocity field $\boldsymbol{u}_{0}$, $2\left(D_{0} \phi_{0} / D t\right)$, can be evaluated. Using $P$ and $\chi,(8)$ gives for the slow variables,

$$
\begin{gathered}
2 \frac{D_{0} \phi_{0}}{D t}=\frac{\sigma_{s 0}\left(D_{0} \sigma_{n 0} / D t\right)-\sigma_{n 0}\left(D_{0} \sigma_{s 0} / D t\right)}{\sigma_{0}^{2}}, \\
\frac{D_{0} \sigma_{n 0}}{D t}=\partial_{y y} P-\partial_{x x} P+2 f \partial_{x y} \chi \quad, \quad \frac{D_{0} \sigma_{s 0}}{D t}=-2 \partial_{x y} P+f\left(\partial_{y y} \chi-\partial_{x x} \chi\right) .
\end{gathered}
$$

Finally, from $2\left(D_{0} \phi_{0} / D t\right), \omega_{0}$ and $\sigma_{0}$, the strain rate of the $\boldsymbol{u}_{0}$ field, a slow quantity $\tilde{r_{0}}$ that identifies the areas of filament ejection can be calculated as in (7) and (10),

$$
\begin{aligned}
& r_{0} \equiv \frac{\omega_{0}+2\left(D_{0} \phi_{0} / D t\right)}{\sigma_{0}} \\
& \tilde{r_{0}}=\frac{\sigma_{0}^{3}}{f^{3}}\left(1-\left|r_{0}\right|\right)
\end{aligned}
$$




\section{B. Filamentation diagnosed from the slow component}

In experiment 2, the slow criterion $\tilde{r_{0}}$ is calculated and shown in Fig.4(b). Both events of filaments ejection are well identified by areas where $\tilde{r_{0}}>0$, even for the secondary filaments modified by the IG waves.

Another check on the theory is based on the fact that in strain dominated regions $(\tilde{r}>0)$, the exponential growth of $|\nabla q|$ characterizing filamentation is associated with an alignment of PV gradient along a specific direction relative to the strain axes. ${ }^{18}$ This preferential direction can be verified via the statistical study of PV gradient alignment for experiment 2. Therefore, two probability density functions (PDF) are calculated in strain dominated regions: the first one concerns the difference between the actual orientation of PV gradient relatively to the compressional axis associated with the slow velocity field $\boldsymbol{u}_{0}$ and its theoretical value, $-\arccos \left(r_{0}\right)$, and the second one concerns the same difference but restricted to the two boxes of Fig. 2. As these PDF, plotted in Fig. 5, present an important peak around zero, there is a clear evidence for the alignment of PV gradient with the theoretical value in strain dominated regions, both in the whole domain and in the two boxes corresponding to the second filaments shedding.

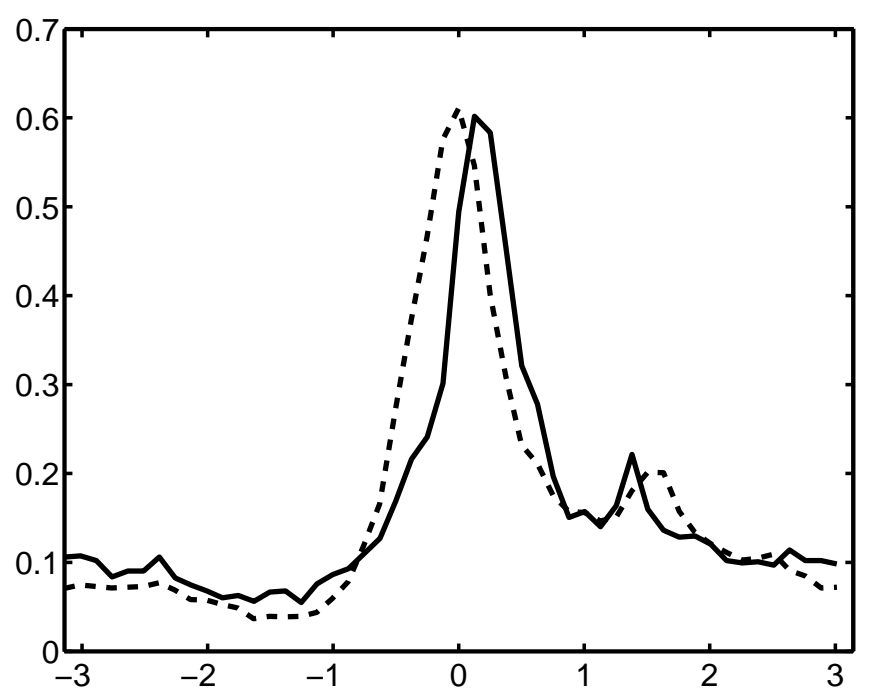

Figure 5: PDF of the difference between the actual orientation of PV gradient relatively to the compressional axis associated with the filtered velocity field $\boldsymbol{u}_{0}$ and its theoretical value after a vortex rotation time scale in the whole domain (dashed line) and in boxes of Fig. 2 (solid line). PDF are calculated for experiment 2 where $\left|r_{0}\right|<1$ and $|\nabla P V|>$ $10 \%|\nabla P V|_{\max }$. 
Figures $4(\mathrm{~b})$ and 5 show that the filtered criterion $\tilde{r_{0}}$ based on the slow component of the flow proves to be a good tool to study vortex erosion by filamentation even when PV is only inverted from geostrophic balance. Many more accurate PV inversion methods than from geostrophic balance exist ${ }^{25,26,27}$. We have tested higher-order inversion, but since the theory of gradient alignment in strain coordinates is not exact ${ }^{18}$ - it neglects in particular the Lagrangian $r$ variations - using more precise balance equations did not improve our results (not shown).

Therefore, PV gradients appear to be in equilibrium with geostrophic dynamics and although the process of filamentation is altered by the interaction between the vortex and IG waves, it can be diagnosed from the modified slow component of the flow which still captures the required information.

\section{Summary}

The purpose of this paper was to study the vortex filamentation process in SW dynamics. Numerical experiments show that this process can be modified by the presence of IG waves irreversibly. In presence of rapid motions, the ejection points of filament are shifted and the SW version of the $r$ criterion $^{18}$ cannot any longer locate them. The filamentation process must be analyzed from the slow component of the flow which, despite its alteration by the presence of IG waves, still captures the required information and we have shown that the $r$ criterion, estimated from the slow quantities, can be used to identify areas of PV filament ejection. These findings are in agreement with the current paradigm that PV advection is dominated by the slow velocity field.

Results presented in this paper pertaining to the filamentation process and a slow $r$ criterion have also been studied in other regimes (not shown) of different Rossby and Froude numbers (from 0.1 to 0.5 ) by modifying the intensity, shape, and size of the vortex and the intensity and wavelength of inertia-gravity waves. They seem to be robust even if PV is only inverted from geostrophic balance.

Further work is needed to quantify the modification of the filamentation process by IG 
waves. We expect it to depend on the intensity and wavelength of the IG waves compared to the intensity and size of the vortex.

\section{ACKNOWLEDGMENTS}

The calculations reported here were done on the NEC SX-5 of the IDRIS (Orsay,

France) (project No. 031589). We thank Guillaume Lapeyre and Patrice Klein for their comments on the manuscript. 


\section{Appendix: Vortex alone experiment}

The difference between the Okubo-Weiss and the $r$ criterions is illustrated in experiment 1. For the $r$ criterion, we consider the $\tilde{r}$ quantity (10) and for consistency, a modified Okubo-Weiss quantity $\lambda=\left(\sigma^{3} / f^{3}\right)(1-|\omega| / \sigma)$ is estimated. Both quantities should be positive in regions of filament ejection. After a vortex rotation time scale, Figs. 6(a) and 6(b), respectively, show the $\lambda$ and $\tilde{r}$ fields, on both figures the gray shading corresponds to the PV anomaly field. They illustrate that the Okubo-Weiss criterion locates filamentation all around the vortex whereas the areas of filament ejection are better identified by the areas where $\tilde{r}$ is positive. This difference is due to the fact that the Okubo-Weiss criterion neglects the rotation of the strain principal axes which is important on the vortex edge and maximal along the ellipse's main axes.

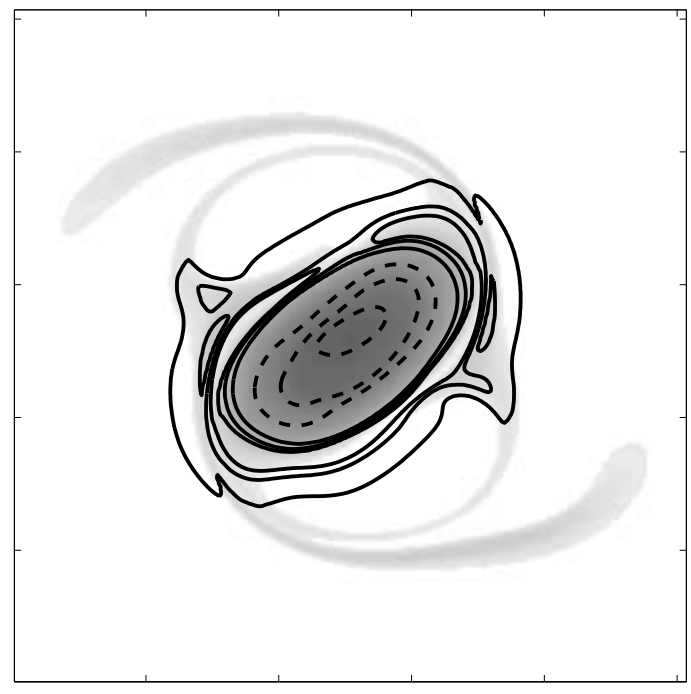

(a)
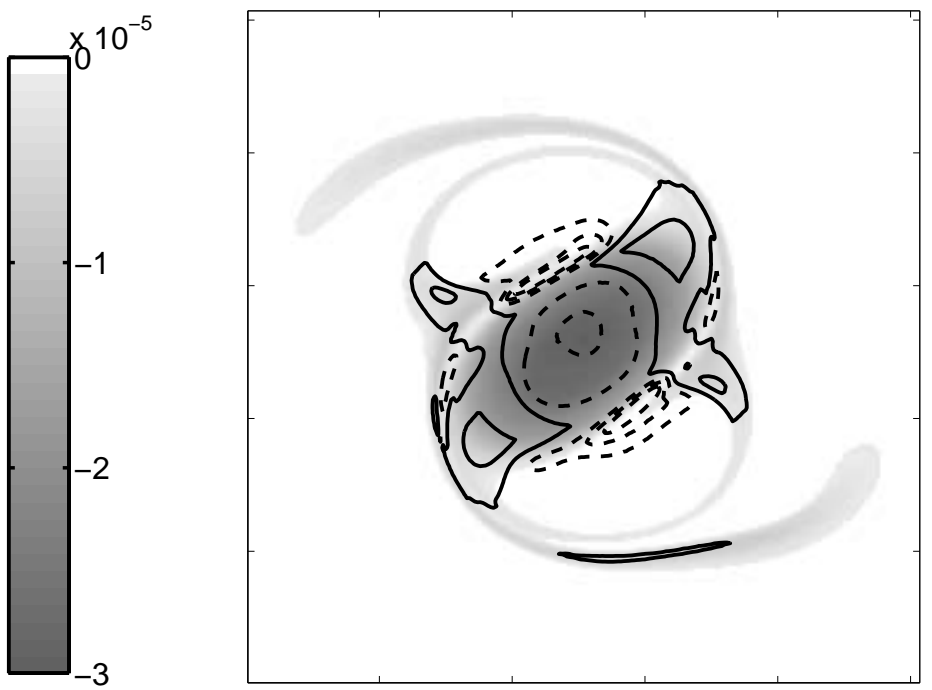

(b)

Figure 6: (a) $\lambda$ field (b) $\tilde{r}$ field after a vortex rotation time scale for experiment 1 . For both figures, solid lines are positive contours and dashed lines are negative contours (interval is $10^{-3}$ ), the gray shading corresponds to the PV anomaly field. 


\section{References}

${ }^{1} \mathrm{~S}$. Kida, "Motion of an elliptic vortex in a uniform shear flow", J. Phys. Soc. Jpn., 50, 3517 (1981).

${ }^{2}$ A. Mariotti, B. Legras, and D.G. Dritschel, "Vortex stripping and the erosion of coherent structures in two-dimensional flows", Phys. Fluids A, 6, 3954 (1994).

${ }^{3}$ D.G. Dritschel, P.H. Haynes, M.N. Juckes, and T.G. Shepherd, "The stability of a two-dimensional vorticity filament under uniform strain", J. Fluid Mech., 230, 647 (1991).

${ }^{4}$ M.V. Melander, J.C. McWilliams, and N.J. Zabusky, "Axisymmetrization and vorticitygradient intensification of an isolated two-dimensional vortex through filamentation", J. Fluid Mech., 178, 137 (1987).

${ }^{5}$ L.M. Polvani, G.R. Flierl, and N.J. Zabusky, "Filamentation of unstable vortex structures via separatrix crossing: A quantitative estimate of onset time", Phys. Fluids A, 1, 181 (1989).

${ }^{6}$ Y. Kimura and J.R. Herring, "Gradient enhancement and filament ejection for nonuniform elliptic vortex in 2D turbulence", J. Fluid Mech., 439, 43 (2001).

${ }^{7}$ W.K. Dewar and P.D. Killworth, "Do fast gravity waves interact with geostrophic motions?", Deep-Sea Res., Part I, 42(7), 1063 (1995).

${ }^{8} \mathrm{P}$. Bartello, "Geostrophic adjustment and inverse cascades in rotating stratified turbulence", J. Atmos. Sci., 52, 4410 (1995).

${ }^{9}$ M. Farge and R. Sadourny, "Wave-vortex dynamics in rotating shallow water", J. Fluid Mech., 206, 443 (1989).

${ }^{10} \mathrm{O}$. Bühler and M.E. McIntyre, "On non-dissipative wave-mean interactions in the atmosphere or oceans", J. Fluid Mech., 354, 301 (1998). 
${ }^{11} \mathrm{O}$. Bühler, "On the vorticity transport due to dissipating or breaking waves in shallowwater flow", J. Fluid Mech., 407, 235 (2000).

${ }^{12}$ A. Okubo, "Horizontal dispersion of floatable particles in the vicinity of velocity singularities such as convergences", Deep-Sea Res., 17, 445 (1970).

${ }^{13} \mathrm{~J}$. Weiss, "The dynamics of enstrophy transfer in two-dimensionnal hydrodynamics", Physica D, 48, 273 (1991).

${ }^{14}$ X. Carton, L. Chérubin, J. Paillet, Y. Morel, A. Serpette, and B. Le Cann, "Meddy coupling with a deep cyclone in the Gulf of Cadiz", J. Mar. Syst., 32, 13 (2002).

${ }^{15}$ S.R. Maassen, H.J.H. Clercx, and G.J.F. van Heijst, "Self-organisation of quasi-twodimensional turbulence in stratified fluids in square and circular containers", Phys. Fluids, 14, 2150 (2002).

${ }^{16} \mathrm{~S}$. Herbette, Y. Morel, and M. Arhan, "Erosion of a surface vortex by a seamount", J. Phys. Oceanogr., 33, 1664 (2003).

${ }^{17}$ B.L. Hua and P. Klein, "An exact criterion for the stirring properties of nearly twodimensional turbulence", Physica D, 113, 98 (1998).

${ }^{18}$ G. Lapeyre, P. Klein, and B.L. Hua, "Does the tracer gradient vector align with the strain eigenvectors in 2D turbulence?", Phys. Fluids, 11, 3729 (1999).

${ }^{19}$ P. Klein, B.L. Hua, and G. Lapeyre, "Alignment of tracer gradient vectors in 2D turbulence", Physica D, 146, 246 (2000).

${ }^{20}$ G. Rivière, B.L. Hua, and P. Klein, "Perturbation growth in terms of barotropic alignment properties", Q. J. R. Meteorol. Soc., 129, 2613 (2003).

${ }^{21}$ D.N. Straub, "Instability of 2D flows to hydrostatic 3D perturbations", J. Atmos. Sci., 60, $79(2003)$.

${ }^{22}$ P. Haynes, Vertical shear plus horizontal stretching as a route to mixing, in Aha Huliko'a Proceedings, U. Hawaii, pp. 73-79, 2001. 
${ }^{23}$ T.Y. Koh and B. Legras, "Hyperbolic trajectories and the Antarctic polar vortex", Chaos, 12, 382 (2002).

${ }^{24}$ R. Bleck and D. Boudra, "Wind driven spin-up in eddy-resolving ocean models formulated in isopycnic and isobaric coordinates", J. Geophys. Res., 91, 7611 (1986).

${ }^{25}$ G.K. Vallis, "Potential vorticity inversion and balanced equations of motion for rotating and stratified flows", Q. J. R. Meteorol. Soc., 122, 291 (1996).

${ }^{26}$ M.E. McIntyre and W.A. Norton, "Potential vorticity inversion on a hemisphere", J. Atmos. Sci., 57, 1214 (1999).

${ }^{27}$ A.R. Mohebalhojeh, "On shallow water potential vorticity inversion by Rossby number expansions", Q. J. R. Meteorol. Soc., 128, 679 (2002).

${ }^{28}$ T.O. Warn, O. Bokhove, T.G. Shepherd, and G.K. Vallis, "Rossby number expansions, slaving principles, and balance dynamics", Q. J. R. Meteorol. Soc., 121, 723 (1995).

${ }^{29}$ D.J. Muraki, C. Snyder, and R. Rotunno, "The next-order corrections to quasigeostrophic theory", J. Atmos. Sci., 56, 1547 (1999).

${ }^{30}$ A.R. Mohebalhojeh and D.G. Dritschel, "Hierarchies of balance conditions for the fplane shallow water equations", J. Atmos. Sci., 58, 2411 (2001).

${ }^{31}$ B.L. Hua, J.C. McWilliams, and P. Klein, "Lagrangian accelerations in geostrophic turbulence", J. Fluid Mech., 366, 87 (1998). 